Les « écoles indigènes » en Algérie à la fin du XIXe siècle : l'expérience de maîtres français et indigènes dans le sud-est algérien au cours des années 1895-1897

\title{
Claude Bisquerra
}

\section{(2) OpenEdition Journals}

\section{Édition électronique}

URL : https://journals.openedition.org/dhfles/2613

DOI : 10.4000/dhfles.2613

ISSN : 2221-4038

Éditeur

Société Internationale pour l'Histoire du Français Langue Étrangère ou Seconde

\section{Édition imprimée}

Date de publication : 1 décembre 2001

ISSN : 0992-7654

Référence électronique

Claude Bisquerra, «Les « écoles indigènes » en Algérie à la fin du XIXe siècle : l'expérience de maîtres français et indigènes dans le sud-est algérien au cours des années 1895-1897 », Documents pour I'histoire du français langue étrangère ou seconde [En ligne], 27 | 2001, mis en ligne le 31 janvier 2014 consulté le 27 mai 2021. URL : http://journals.openedition.org/dhfles/2613 ; DOI : https://doi.org/ $10.4000 /$ dhfles. 2613 


\title{
Les « écoles indigènes » en Algérie à la fin du XIXe siècle : l'expérience de maîtres français et indigènes dans le sud-est algérien au cours des années 1895-1897
}

\author{
Claude Bisquerra
}

1 L'absence de politique suivie et de plans définis des trois régimes successifs en Algérie depuis l'arrivée des Français (Monarchie de Juillet, Seconde République et Second Empire) avait voué à l'insuccès cinq tentatives de réformes scolaires. Après plusieurs enquêtes sur tout le territoire, la réorganisation de l'enseignement primaire des indigènes devient effective avec le décret du 13 février 1883, modifié par celui du 9 décembre 1887, appliquant à l'Algérie, avec quelques modifications indispensables, les dispositions en vigueur en Métropole.

2 La tâche était d'envergure. Elle concernait plusieurs domaines et comportait, en dehors de l'usage du français comme langue commune, des programmes et des méthodes spécifiques pour les indigènes. J'ai jugé intéressant de chercher à définir les structures utilisées, les enseignants concernés et comment les instituteurs de ces écoles isolées qui, par leur position géographique et leur administration militaire particulières reflètent ce qui me semble être " la situation extrême » de l'influence scolaire de la langue française dans ce pays, appliquaient les directives du Ministère de l'Instruction publique pour la réalisation de ce dessein. Des documents manuscrits constituent le corpus de cette étude ${ }^{1}$. Ils sont relatifs à deux types $\mathrm{d}^{\text {' }}$ écoles indigènes ${ }^{2}$ » spécialement créées pour les autochtones : l'école sédentaire de l'oasis de Négrine $^{3}$ et les écoles nomades des tribus des Allaouna et des Brarcha qui dépendaient administrativement du cercle militaire de Tébessa. Datés de 1895 à 1897, ils émanent, pour la plupart, de l'instituteur français de Négrine, mais également de trois adjoints et moniteurs indigènes des écoles nomades et, en moindre part, de fonctionnaires de l'Académie du 
Département de Constantine et de chefs militaires. Ces documents - quelque 125 lettres et rapports à caractère officiel et personnel -, plus témoignages de vies qu'exposés théoriques, couvrant une période limitée, ne peuvent donner qu'un aperçu subjectif, reflet de personnalités différentes, d'une étape spatialement et temporellement déterminée de l'histoire de l'enseignement du français en Algérie.

\section{Programmes, méthodes et moyens pour la scolarisation}

3 La période examinée se situe sous le Rectorat Jeanmaire, après l'élaboration et l'application du Plan d'études et programmes de l'enseignement primaire des indigènes rédigé en 1890, après le décret du 18 octobre 1892 réorganisant l'enseignement indigène public et privé, " véritable charte de l'enseignement des indigènes en Algérie " (Besserve-Bernollin, etc. 1981 : 47), mais avant le remaniement de ce même Plan en 1898, ces deux plans successifs étant considérés comme «le progrès le plus décisif qui ait été accompli depuis 1830 » (Mirante cité par Ageron 1968 : 537). Malgré les principes égalitaires chers à la République, se dessine à la fin du XIXe siècle une nouvelle conception de l'enseignement du français pour les non-francophones (Spaëth 1999). L'ensemble des programmes fut jugé trop complexe autant pour les écoliers indigènes que pour leurs maîtres (Plan 1890). On renonça alors à appliquer pour l'enseignement des indigènes les mêmes critères adoptés en Métropole et l'on eut soin d'exclure des programmes toutes les curiosités grammaticales ou historiques ${ }^{4}$. L'Arabe ne devait plus être considéré " comme un phonographe enregistrant des mots dont il ne comprenait pas la signification » (Poulard 1910 : 183), mais on voulait s'adresser à son intelligence, l'habituer à observer, à raisonner, éveiller sa curiosité. Ces principes s'inscrivaient en opposition avec l'enseignement traditionnel coranique, figé dans une routine scolastique. La langue française, « instrument de nos échanges et véhicule de nos idées » (B.E. 1897 : 187), est à la base de l'instruction des indigènes. Dès le cours préparatoire, jugé comme le plus important des trois cours, celui qui reçoit le plus grand nombre d'élèves et demande le plus de travail, le programme du Plan insiste sur l'importance des exercices de langage qui restent «à tous les degrés de l'école indigène, le pivot de l'enseignement » (Plan 1890 : VII) puisqu'ils font accéder le petit indigène au nouveau code sémantique. En dehors de la lecture ${ }^{5}$ qui occupe cinq heures par semaine sur un total de $30 \mathrm{~h}$, la Commission du Plan avait réservé 9 heures hebdomadaires aux exercices de langage, car

c'est, en effet, par son degré le plus élémentaire que l'école indigène diffère le plus de l'école qui reçoit des Français. (Plan 1890 : VII).

4 La priorité est donnée à l'implantation des écoles. La phase d'expansion qui culmine entre 1892 et 1898 fait suite à un effondrement de 1889 à 1891 et sera suivie d'une rechute de 1899 à 1901. La réforme envisageait l'ouverture de 60 à 62 écoles indigènes à deux classes, d'environ 50 élèves chacune, chaque année sur tout le territoire de l'Algérie. Dès sa création en 1892, l'école de Négrine est citée en exemple ${ }^{6}$ et en 1896 , le commandant supérieur militaire notait qu'il restait encore une somme sur le crédit alloué en 1895 et que celui de 1896 était intact (10.1.1896) ${ }^{7}$. Par la facilité de leur installation et la rapidité des progrès réalisés par les élèves, les écoles nomades semblaient destinées à une très grande extension ${ }^{8}$. Les dépenses pour le mobilier du maitre et des élèves grevaient peu le budget : 600 fr pour la tente de l'école, procurée 
par les tribus, $20 \mathrm{fr}$ une table-pliante pour le bureau du maître, 50 fr pour 20 nattes pour les élèves, 100 fr pour 20 cartables en bois pour écrire (27.10.1896), une pioche pour creuser les rigoles autour de la tente pendant l'hiver (13.11.1896), des piquets en fer « bien solides pour fixer la tente en cas de tempête » et des cordes « en poils de chèvre et laine suivant la fabrication des indigènes nomades ${ }^{9}$ " (9.4. 1896). Les faibles populations de Négrine et des écoles nomades ne requièrent qu'une école préparatoire à une classe, puis en 1894, à deux classes pour Négrine ${ }^{10}$ (B.E. février 1895).

\section{Nature, formation et qualification des enseignants}

5 La nomination d'un instituteur français pour l'école sédentaire répondait au souci de confier l'éducation des indigènes à celui que l'on considérait comme le dépositaire non seulement de la vraie connaissance de la langue française, de la pédagogie et de la culture mais encore de l'idéologie laïque, patriotique et civilisatrice de la France. En revanche, la nomination d'adjoints ou moniteurs indigènes pour les écoles nomades répondait avant tout au désir d'inspirer confiance aux populations itinérantes non familiarisées avec l'européen afin d'obtenir plus facilement la scolarisation régulière de leurs enfants. De plus, seuls les indigènes pouvaient accepter d'être nommés dans des endroits où " jamais ne se résignerait à vivre un instituteur français même avec un traitement élevé » (Plan 1890 : VI). En tant qu'adjoints, ils possédaient le brevet élémentaire, obtenu après trois ou quatre années de préparation dans les cours normaux spéciaux ${ }^{11}$. Deux des maîtres des écoles nomades sortaient du cours normal de la Bouzaréa d'Alger ${ }^{12}$. Au dessous de cette catégorie, les maîtres étaient moniteurs, comme M. Labrache, en possession du certificat d'études indigène, le diplôme nouveau spécialement créé pour les indigènes par le décret de 1883, sanctionnant les quelques années d'étude à l'école primaire. M. Ben Ali participera en août 1897 à un voyage d'étude de quinze jours en France, auquel avaient droit les maitres indigènes pourvus du brevet élémentaire comme prime d'encouragement à accéder à un niveau d'instruction plus élevé et pour leur permettre de connaître la Métropole (13.7.1897) ${ }^{13}$. Il oubliera un moment « le froid si rigoureux de Chréia pendant les froides nuits d'hiver » (16.1.1897), son grand isolement et bien d'autres difficultés matérielles qu'il décrit au commandant :

Sans parents ni alliés, je n'ai que vous qui me remplacez tout, je ne me plains qu'à vous. Je ne peux pas faire la cuisine et la classe, je ne peux laver mes habits non plus. Tout seul je ne peux rien faire et mon traitement ne me permet pas d'avoir quelqu'un (4.12.1896).

En l'absence d'école principale proche, M. Labrache fait office de directeur et signe « Le moniteur indigène chargé de la Direction de l'école des Allaouna » (31.12.1896). Malgré " (leur) séjour, dépourvu de charmes, dans (leur) oasis » (15.3.1895), l'instituteur français de Négrine, secondé par sa femme, adjointe, restera à son poste 14 ans ${ }^{14}$. En revanche, M. Medouhès demande à quitter l'école nomade un mois après sa nomination, déclarant, comme le réfère l'inspecteur primaire de Batna au commandant,

qu'il ne pouvait se charger d'enseigner la langue arabe, qu'il était peu satisfait de la vie nomade et qu'il ne voulait pas coucher sous la tente $(30.10 .1896)^{15}$.

7 En général, l'Arabe devenait enseignant plus par nécessité que par choix ${ }^{16}$. Le refus d'enseigner la langue arabe, s'il peut être dicté par la volonté de ne pas empiéter sur le domaine religieux du taleb, va étrangement dans le sens de la politique dite «des 
colons ", hostiles à la scolarisation des indigènes, qui voyaient dans l'enseignement de cette langue le moyen de compromettre l'influence française en Algérie. Son remplaçant, M. Ben Ali était sujet français mais non naturalisé, la naturalisation n'étant pas obligatoire pour enseigner dans les écoles indigènes (16.1.1896). Les instituteurs indigènes possèdent une complète maîtrise $d u$ français écrit, leur calligraphie est parfaite et leurs lettres ne contiennent aucune erreur de syntaxe ou grammaire, seulement quelques fautes d'orthographe. Mais, malgré leur formation particulière et le profond attachement à la France témoigné par M. Ben Ali dans une lettre au commandant :

Je suis votre fils et celui de la France. Si je suis ici loin de mon pays et des miens, c'est pour contribuer, dans ma modeste part, à répandre ses idées civilisatrices (14.12.1896),

adjoints et moniteurs indigènes étaient soupçonnés de ne pas avoir complètement assimilé et, par conséquent, de ne pas pouvoir diffuser "les idées françaises », de manquer de compétences pédagogiques se traduisant par des défauts dans la manière d'enseigner. M. Testas accomplit sa tâche avec un sens aigu du devoir : pendant les vacances de Pâques, il renonce à un séjour à Tébessa, qui aurait amélioré son état de santé, car il considère ce « brusque départ comme une fuite » (19.3.1896) et ne veut pas s'absenter pour veiller à ce que les prescriptions du capitaine " soient bien exécutées " $(31.3 .1896)^{17}$.

\section{Rôle des militaires}

Il existait, note déjà Berthelot en 1887, une sympathie naturelle entre armée et instruction publique (B.U. 1887). Presque toutes les lettres des instituteurs sont adressées au commandant ou au capitaine, chefs des bureaux arabes, ces organismes militaires chargés du gouvernement des populations musulmanes de l'Algérie, qui tenaient à la fois du service de renseignement, du contrôle et de l'administration proprement dite (D.a. 1947). La dispersion des écoles, surtout des écoles nomades, constituait un problème pour le contrôle de leur fonctionnement. En raison de la difficulté à joindre les deux écoles nomades au cours de leurs fréquents déplacements dans les vastes étendues, le Recteur les soumet, « en outre des visites de l'inspecteur primaire " (14.10.1896), à la surveillance du commandant supérieur militaire et non à celle de l'instituteur français, comme le stipulait le décret du 18 octobre 1892:

Je compte sur le bienveillant concours de l'autorité militaire pour en assurer la marche régulière. J'espère que les officiers voudront bien visiter ces écoles et rendre compte, dans leurs tournées, de leur bon fonctionnement (26.11.1896).

Quant à M. Ben Ali, il ne manque pas de remercier le capitaine de l'intérêt qu'il porte à son école ${ }^{18}$.

11 Par leur meilleure connaissance de l'âme musulmane et leurs méthodes moins arbitraires que celles des civils, les militaires étaient mieux acceptés par les populations. 


\section{Rapports entre la population indigène et l'école / la religion}

12 Chaque mois, les instituteurs envoyaient le tableau de la fréquentation des classes. Sur les 277 enfants de Négrine recensés par le cheikh, M. Testas compte entre 58 et 62 écoliers inscrits dans les deux classes. Les effectifs des écoles nomades sont plus réduits : entre 11 et 24 élèves fréquentant la classe ${ }^{19}$, même si M. Labrache envisage un nombre nettement supérieur:

Si la tribu venait à mettre de la bonne volonté à envoyer ses enfants à l'école, notre école comptera plus qu'une cinquantaine ou soixantaine d'élèves (8.3.1897).

13 D'après les quelques tableaux des fréquentations relevés dans les lettres, il semble que les périodes les plus favorables pour les demi-jours de classe réellement faits soient décembre pour les deux types d'écoles et mars (écoles nomades) / avril ou mai (Négrine) ; pour la présence effective des élèves, de nouveau décembre pour les deux types d'écoles et février (Négrine, sauf en 1895 à cause de l'épidémie de coqueluche) / mars (écoles nomades). La présence effective diminue de manière significative à partir d'avril. Les facteurs qui ont une influence sur le fonctionnement des écoles semblent concerner pareillement élèves et maitres, mais si l'offre didactique reste élevée à l'approche de l'été, les élèves désertent les classes.

14 La religion est l'un des obstacles au bon fonctionnement de l'école française depuis ses débuts. L'enseignement laïc est ressenti par les musulmans comme " facteur dépersonnalisant " (Sekfali 1993 : 4). Ils craignent qu'elle ne fasse oublier à leurs enfants la religion islamique et ses règles connues (Mérad 1963). Le maître indigène se heurte davantage à l'hostilité de ses coreligionnaires car il est l'incarnation du pouvoir de la nation dominatrice et fait figure de renégat. La fréquentation de l'école était au centre des préoccupations des maitres et de l'instruction publique ${ }^{20}$. La raison de la mauvaise volonté des indigènes à envoyer leurs enfants à l'école est, pour M. Labrache, essentiellement d'ordre religieux : alors que la fréquentation de l'école est régulière pour le mois de décembre, il n'a relevé qu'une seule absence, significative, celle du fils du taleb pour " cause de fanatisme » (31.1.1897). Grâce à ses bonnes paroles et au conseil qu'il donne au taleb de rapprocher sa tente de celle de l'école de manière que les enfants fréquentant l'école coranique aient moins de chemin à parcourir pour répondre à l'appel de l'école française, ce dernier consent à camper à une dizaine de mètres et à ramener son fils. Ce passage montre, dans ce cas, que la liberté confessionnelle des musulmans était respectée et que, parallèlement à l'institution française, l'institution musulmane continuait à dispenser son enseignement. En plus de l'influence des maitres, les autorités françaises recherchent la collaboration des cheikhs et des caïds dont elles connaissaient l'ascendant sur les populations. Le commandant note à l'adresse du caïd des Allaouna et des Brarcha:

Veuillez porter à la connaissance de vos administrés que des écoles nomades sont installées dans votre tribu et que l'autorité serait désireuse de voir les écoles fréquentées par le plus grand nombre d'élèves possible. L'instruction qui y est donnée est française et arabe. Ils ont donc tout intérêt à y envoyer leurs enfants (29. 1. 1896).

15 Aussi M. Testas déplore-t-il la mort du cheikh de Négrine,

véritable perte pour nous et pour notre école dont il assurait la fréquentation d'une manière remarquable [9.3.1895) 
et recommande-t-il vivement au commandant de faire en sorte que son successeur agisse de même (9.3.1895). Le cheikh et le caïd facilitent encore l'implantation des écoles nomades en se chargeant de la fourniture des matériaux nécessaires à la fabrication du matériel, effectuée par les membres de la tribu (21.7.1896). M. Ben Ali peut écrire :

Pour le moment, je n'ai qu'à faire des compliments et à féliciter le caïd Seghir dans mes rapports à mes chefs et autre autorité scolaire (31.12.1896) ${ }^{21}$.

17 Plus que la personnalité du maître dont on voulait qu'il soit « le second père " pour ses élèves (Plan 1890 : 123), c'est son image associée à la puissance de la France qui pâtit de l'hostilité dont il est l'objet. Le discrédit dont souffre l'instituteur rejaillit sur l'école française dans son ensemble. Bien qu'elle semble dérisoire, la requête du maitre des Allaouna d'un trépied qui lui éviterait de s'asseoir par terre - comme il consent à le faire devant ses élèves durant la classe - pour recevoir les honorables visiteurs est révélatrice de l'importance qu'accordaient les indigènes au respect de la hiérarchie des fonctions (3.6.1897).

\section{Conditions d'enseignement}

Les autres obstacles au bon fonctionnement de l'école sont les conditions géographiques, climatiques et de vie. La température de la cour de l'école de Négrine atteint, en mars, $40^{\circ}$ au soleil à $9 \mathrm{~h}$ du matin, et, en général, écrit $M$. Testas,

dès le mois d'avril, la température devient excessive et pendant tout le mois de mai l'atmosphère n'est plus respirable. A cette époque éclosent toutes les maladies adhérentes au climat, la population est décimée par les fièvres (20.1.1896).

Les absences sont alors nombreuses. Elèves et maîtres ont leur santé minée par les fièvres, les maladies ou les épidémies ${ }^{22}$. L'école doit être fermée pendant des travaux de réfection (2.3. J895) et pour la désinfectation des classes après l'épidémie de coqueluche $(1.5 .1895)^{23}$. Les grandes vacances étaient fixxées par le Recteur du 1er juin au 30 septembre pour Négrine, du 1er juin au 31 août pour les écoles nomades, avec un supplément de vacances à ces dernières du 1er décembre au 28 février, « au moment où le recrutement des élèves pouvait difficilement avoir lieu » (5.5.1897). L'invasion des criquets et la moisson des orges monopolisent les bras valides, y compris ceux des enfants scolarisés. M. Testas, conscient d'être investi d'un rôle défini à l'époque comme celui d'« un agent général de civilisation élémentaire plutôt qu'un maître d'école au sens ordinaire du mot " (D.a. 1947 : 26), conduit alors lui-même ses élèves sur les chantiers de destruction des acridiens et de leurs œufs (1.5.1897). Dans une autre lettre, il requiert la construction d'un hangar dans la cour de l'école pour éviter de faire dormir dans la salle de classe le gardien, soupçonné d'être à l'origine du vol de menus objets scolaires (10.1.1896). En ce qui concerne les écoles nomades, les fréquents déplacements des smalas, tous les trois mois environ, ne facilitent pas l'envoi du matériel, acheminé sur des pistes, à dos de cheval, de chameau et sur des caravanes de passage.

Encore moins la régularité des cours : le maître déplore la perte de

quelques-uns de (ses) meilleurs élèves qui ont quitté l'école pour rester avec leurs parents auprès du khalifa du caïd (31.1.1897) ${ }^{24}$.

21 Le commandant signalait à l'inspection primaire de Batna que M. Medouhès n'avait toujours pas commencé la classe le 10 novembre, le matériel et les fournitures scolaires n'étant pas encore arrivés (10.11.1897) ${ }^{25}$. Dans ces conditions, la bibliothèque scolaire, « 
ce complément utile des leçons du maître » (B.E. janvier $1896: 30$ ), dont la création est recommandée partout, ne peut être constituée (26.10.1896).

\section{Manuels scolaires et méthodes d'enseignement du français}

Les lettres des maitres contiennent les listes de commande de livres scolaires ${ }^{26}$ pour les différentes matières enseignées qui comprenaient, en outre, la langue arabe, l'agriculture, les travaux manuels et les leçons d'observation. Voici les titres des manuels de français tels qu'ils sont transcrits - incomplètement - par les maîtres : pour M. Testas : Alphabet, Regimbeau (20 ex.), L'Année enfantine de lecture, Guyau (12 ex.), Grammaire de l'enfance, Leclair et Rouzé (20 ex.), 250 dictées cours élémentaire, Luptil (1 ex.), Méthode d'écriture en 11 cahiers. Cahiers d'écriture, Flament, Les premières lectures enfantines (10 ex.). Pour M. Labrache : Livre de langage, Scheer (1 ex.), Méthode de lecture, Cuissart (5 ex.), Méthode de lecture, Lacabe (20 ex.), Méthode de langage et de lecture. Carré, Année préparatoire de grammaire, Larive et Fleury.

Le choix d'une méthode de lecture était laissé à l'instituteur (Plan 1890). Les deux maitres utilisent les manuels les plus répandus, objets de plusieurs rééditions, dont la publicité apparaissait dans le B.E., cette revue pédagogique fondamentale qui " traduisait concrètement l'esprit du Plan d'études en pratique pédagogique " (BesserveBernollin, etc. 1981 : 134). Le B.E. prodigue des conseils extrêmement précis pour toutes les matières et relate les expériences des instituteurs ${ }^{27}$. Les listes citent le manuel d'I. Carré qui préconisait la méthode directe, dite maternelle - c'est-à-dire celle que la mère utilise spontanément pour apprendre à parler à son enfant - sans avoir recours à la traduction, sauf nécessité majeure, en se basant sur sa propre expérience avec les petits Bretons de Basse Bretagne pour qui l'ignorance de la langue française était aussi totale que pour les petits indigènes (Carré 1891, Spaëth 1999). Cette méthode qui habituait l'enfant à penser directement en français était une innovation. Par ailleurs, on remarque que, contrairement à $\mathrm{M}$. Testas dont la liste trahit des exigences traditionnelles de l'enseignement, dictées par le niveau supérieur de sa classe, avec la prépondérance de la grammaire, des dictées, de l'écriture, l'instituteur indigène fait une large part à la lecture et surtout au langage, reflétant ainsi l'autre spécificité de l'enseignement pour les indigènes ${ }^{28}$. On aurait souhaité trouver, dans les lettres, des informations sur les critères qui ont déterminé le choix des manuels de la part des maitres et le genre de difficultés rencontrées avec leurs élèves. Malgré leur souci de s'adapter aux spécificités des élèves indigènes, les manuels laissaient encore beaucoup à désirer. Le Premier livre de lecture à l'usage des écoles et des indigènes des colonies de Carré est critiqué, en 1898, dans une note de l'Académie d'Alger :

il peut fournir tout au plus des sujets de langage. Comme livre de lecture, il a, peu s'en faut, les mêmes défauts pour les indigènes que les petits livres de Bruno, de Guyau, de Jost et Humbert; il n'en a pas leurs qualités (B.E. août 1898 : 118).

Les sujets de lecture semblent, de prime abord, bien appropriés - école, famille, enfant, etc. -, l'exposition en est claire, les gravures attrayantes et l'on peut conserver la méthode préconisée consistant « à faire du thème de la leçon de lecture, le thème d'un petit exercice de langage préalable » (B.E. août 1898 : 118) mais les descriptions des objets tels que matelas, lampadaire, divan, piano et autres proposent des modèles inconnus aux élèves. Le livre ne s'adresse pas du tout aux indigènes, malgré les 
promesses du titre ${ }^{29}$. Deux lettres font mention de résultats. M. Ben Ali fait part au capitaine, avec grande satisfaction, des progrès de ses élèves et, dans le but de les encourager à persévérer, lui demande de lui envoyer

de quoi contenter ce petit monde zélé, tels que bons points, tableaux d'honneur, croix scolaire etc. (13.5.1897).

Quant à M. Testas, il est fier d'envoyer un cahier de ses élèves au commandant afin qu'il se fasse

une idée du degré d'instruction où (ils) sont arrivés et les progrès réalisés par eux (12.5.1896).

Il s'agit du cahier de dessin de ses meilleurs élèves dans les travaux utiles de l'atelier de menuiserie, effectués pendant l'année $1895-96^{30}$. Dans l'esprit de la réforme, l'enseignement pratique a l'avantage de favoriser l'enrichissement de la langue par le concret. S'il n'est pas possible de juger de la réalisation des objets dessinés, nous pouvons en revanche, grâce à la légende qui accompagne les dessins, apprécier la qualité de la calligraphie des élèves, presque aussi sûre, pour certains, que celle de leur maître, et la variété du vocabulaire technique utilisé (Fig. 1, 2). L'enseignement pratique atteint plusieurs objectifs à la fois : pédagogique, éducatif, politique et utilitaire, ce dernier plus tangible que celui de l'enseignement de la langue française. Tout le monde tire profit des réalisations de l'atelier : la classe entière - il restait un spécimen de chaque exercice à l'école - et la population indigène adulte qui apprennent, grâce à la barrière faite pour clôturer la cour, la sacralisation de l'espace de l'école et la nécessité d'une séparation entre humains et animaux, par hygiène élémentaire et tranquillité sonore ; le maitre qui se fait faire - selon ses désirs et rapidement - un meuble pour ses besoins personnels ; enfin le caïd et quelques élèves qui ramènent chez eux - ces derniers en avaient le droit - un meuble utilitaire, avec lequel pénètre dans les foyers indigènes la conception occidentale de bien-être et de progrès ${ }^{31}$.

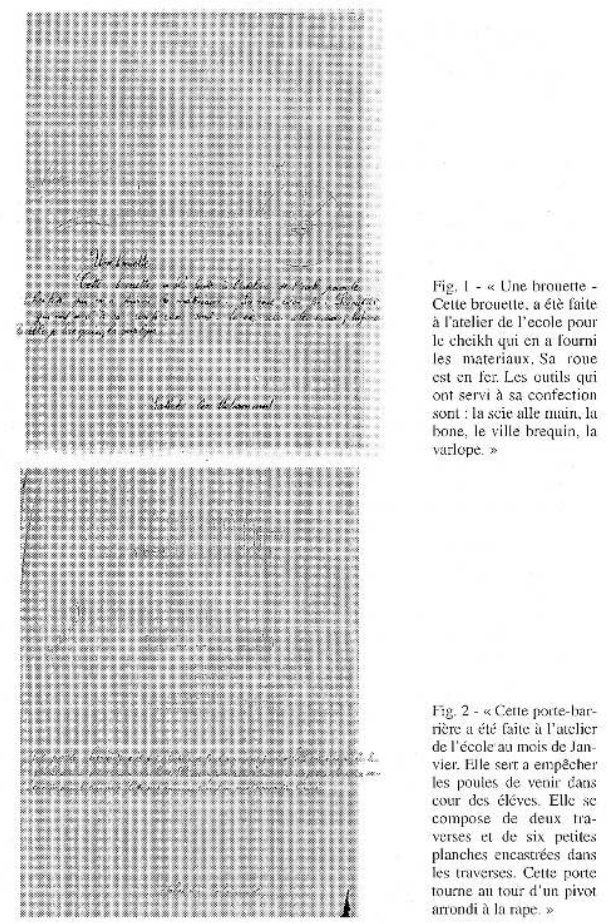




\title{
Conclusion
} accomplissent leur tâche avec sens du devoir sans toutefois se poser de questions sur les fondements sur lesquels ils reposent. A aucun moment n'apparaissent, chez le maître français comme chez les maîtres indigènes, de doutes sur la validité de l'entreprise, la justesse et la nécessité d'enseigner aux indigènes non seulement la langue mais encore la civilisation françaises et l'idée de progrès qui y est attachée, selon une vision ethnocentrique propre à la France à cette époque, qui semble partagée par l'un des maîtres indigènes. Aussi ne trouve-t-on jamais de jugements normatifs sur la politique de scolarisation de la France en Algérie qui divisait pourtant, à l'époque - et continue à diviser, surtout depuis l'indépendance -, hommes politiques, historiens, critiques et grande partie de l'opinion publique ${ }^{32}$. On ne peut s'empêcher de regretter l'absence de témoignages sur une éventuelle contribution de l'institutrice à la diffusion $\mathrm{du}$ progrès dans des domaines typiquement féminins, tels l'hygiène, la nutrition, les soins médicaux.

Loin de la France et isolés culturellement de son influence, les maîtres français et indigènes semblent davantage être aux prises avec les difficultés quotidiennes - réussir à faire la classe et au plus grand nombre d'élèves possible - qu'avec des problèmes spécifiquement didactiques. Bien que le rôle des instituteurs soit déterminant pour le succès de l'entreprise, il est certain que la réalisation et l'application des nouvelles mesures du Plan dépendent aussi, pour beaucoup, des conditions particulières dans lesquelles ils exercent. Les militaires influent notablement sur les questions administratives et matérielles concernant les enseignants et sur leurs rapports avec les indigènes.

L'attention se porte sur les moyens et les méthodes, comme les choix des manuels, les exercices de travaux pratiques, valorisés ${ }^{33}$, et l'enseignement, dès l'école primaire, de l'arabe vulgaire parlé mais également écrit, dans le but, semble-t-il, d'appliquer à cette matière les mêmes méthodes rationnelles utilisées pour les autres puisque seul le français que l'on voulait faire apprendre à tous devait permettre la communication et la compréhension entre les deux peuples.

À la lecture de ces lettres, il résulte que les problèmes les plus immédiats à résoudre par les maîtres sont la fréquentation de l'école et l'enseignement d'une langue qui ne soit pas éloignée de la réalité indigène quotidienne et il apparaît évident, comme l'écrit l'instituteur français, que le second conditionne le premier car

ce n'est que quand ils verront le résultat immédiat de l'instruction que l'on finira par avoir des élèves fréquentant l'école française avec bonne volonté (4.2.1895).

\section{BIBLIOGRAPHIE}

\author{
Sources manuscrites
}

Documents pour l'histoire du français langue étrangère ou seconde, 27 | 2001 
Centre des Archives d'Outre-mer, Aix-en-Provence, F 80 k / 22 / 10-11.

Sources imprimées

AGERON, Charles-Robert (1968) : Les Algériens musulmans et la France (1871-1919). 2 vol. Paris, P.U.F. BESSERVE-BERNOLIN, Lucette - CARBUCCIA, Georges-Henri - CONYGEARE-GRÉZEL, Emilienne HAZAN, Emile - SAURIER, Henri (1981) : 1830-1962 : des enseignants d'Algérie se souviennent... de ce qu'y fut l'enseignement primaire. Toulouse, Privat.

Bulletin de l'enseignement des indigènes de L'Académie d'Alger (B.E.).

Bulletin universitaire de l'Académie d'Alger (B.U.).

CARRÉ,Irénée (1891) : « De la manière d'enseigner les premiers éléments du français aux indigènes de nos colonies et dans les pays soumis à notre protectorat » [Extrait de la Revue Pédagogique, 15 mai 1891]. Paris, Delagrave.

COLONNA, Fanny (1975) : Instituteurs algériens, 1883-1939. Paris, Presses de la Fondation nationale des Sciences Politiques.

Documents algériens (D.A.), nº 7, juin 1946.

Documents algériens, $n^{\circ}$ 11, 8 décembre 1947.

Documents algériens, $\mathrm{n}^{\circ} 12,15$ décembre 1947.

JOANNE, Adolphe (1893) : Géographie de l'Algérie. Paris, Hachette et C $C^{\text {ie }}$.

LÉON, Antoine (1991) : Colonisation, enseignement et éducation. Etude historique et comparative. Paris, L'Harmattan.

MACHUEL, Louis (1885): Méthode de lecture et de langage à l'usage des étrangers de nos colonies. Préface. Paris, A. Colin et $\mathrm{C}^{\text {ic }}$. Alger, Jourdan.

MERAD, Ali (1963) : « Regard sur l'enseignement des Musulmans en Algérie, 1880-1960 », Confluent, 32-33, 596-646.

MOURLAN, Pierre (1903) : Législation et réglementation de l'enseignement primaire public des indigènes en Algérie. Rôle de l'école dans la colonisation, Thèse de l'Université de Poitiers. Faculté de droit. Dijon, Union typographique.

Plan d'études et programmes de l'enseignement primaire des indigènes (1890). Paris, Hachette, C. Delagrave.

POULARD, Maurice (1910) : L'enseignement des indigènes en Algérie. Alger, impr. Gojosso.

RAMBAUD, Alfred (1892) : L'enseignement primaire des Indigènes musulmans d'Algérie et notamment dans la Grande Kabylie. Paris, Delagrave.

SALEMA, Maria José - KAHN, Gisèle -TEIXEIRA, Luis Felipe (1999) : L'enseignement de la langue et de la littérature françaises dans la seconde moitié du XIX ${ }^{e}$ siècle. Actes du Colloque de la SIHFLES organisé à Sintra-Portugal du 1er au 3 octobre 1998, Documents pour l'histoire du français langue étrangère ou seconde 23, juin 1999.

SEKFALI, Abderrahim (1993) : Les maîtres de l'école primaire de l'enseignement public dans le Département de Constantine, de 1890 ci 1939, Thèse de Doctorat d'État. Aix-en-Provence.

SPAËTH, Valérie (1999) : « La didactique du français pour étrangers dans la seconde moitié du XIX siècle : une comparaison des méthodes destinées aux Européens, aux patoisants, aux colonisés » in SALEMA - KAHN- TEIXEIRA éds. (1999), 124-140. 
TURIN, Yvonne (1971) : Affrontements culturels dans l'Algérie coloniale : écoles, médecine, religions. 1830-1880. Paris, Maspéro.

\section{NOTES}

1. CAOM F $80 \mathrm{k} / 22$ / 10-11.

2. Ces écoles remplacèrent celles que l'on appelait, suivant l'époque et les régions, « arabesfrançaises », « maures-françaises », « kabyles-françaises » (Poulard $1910: 100)$.

3. Dans le sud-est algérien, près de la frontière tunisienne, à quelque $150 \mathrm{~km}$ au sud-ouest de Tébessa.

4. Certains reprochaient à ces programmes simplifiés, privilégiant l'enseignement pratique, de favoriser la formation d'une main-d'œuvre qualifiée pour les Européens ; leurs partisans répondaient : «L'essentiel est d'apprendre le français aux générations qui grandissent. Qu'on fasse simplement d'abord, plus tard on fera mieux » (Carré $1891: 25$ ).

5. Parmi les livres de classe dont l'achat incombait aux communes, seul le livre de lecture était obligatoire (B.E. février 1896).

6. « (elle) mérite, sous plusieurs rapports, une mention spéciale parmi les écoles indigènes : la fréquentation y est parfaite. Tous les élèves inscrits, 52 , viennent régulièrement en classe. L'école occupe une belle position sur un plateau, la salle de classe en est spacieuse, élevée, bien aérée. Le logement du moniteur, très bien installé, comprend deux pièces plus la cuisine. Prix de revient : seulement 5.000 francs. Le génie militaire chargé du travail, a procédé par réquisitions et n'a employé, à part les ouvriers du génie, que la main-d'œuvre indigène. Ce système est à imiter » (B.U. avril $1892: 182$ ).

7. «Entretien école Négrine : 150 crédit 1895 encore intact. Achat et entretien de l'école de Négrine et du mobilier de l'instituteur : $200 \mathrm{fr}$, reste 52 fr sur crédit de 1895, crédit 1896, intact ». M. Testas fait des économies : pour la demande de bois de la menuiserie, il précise au commandant que « les matériaux serviront à faire des objets d'utilité pour les indigènes qui en solderont le prix, ce qui permettra de renouveler la provision de bois sans avoir à nouveau recours à votre budget » (3.10.1896).

8. « Nulle part, l'école n'occupe une aussi grande place que dans la vie de ces tribus vagabondes (...). Au centre du campement, une grande tente se dresse, plus haute et plus spacieuse que les autres ; c'est l'école. Les élèves y sont assis en rond sur des nattes, les jambes croisées, un tableau de lecture et un cahier sur leurs genoux. Les leçons ont lieu, comme dans nos écoles, par divisions basées sur l'âge (...). La tribu quitte son campement dès que les pâturages alentour sont épuisés. Les écoliers arrachent les piquets de la tente qui sert d'école, roulent la toile et placent le tout sur le dos d'un chameau, Cinquante, soixante km plus loin, on s'arrête, on plante de nouveau les piquets, on dresse la tente et les leçons recommencent, sans autre interruption que le voyage de la veille (...). L'expérience est faite : les écoles nomades ne sont plus une nouveauté ; elles sont une révélation de notre pouvoir » (B.U. avril 1892 : 312-313). Pourtant, certains les ridiculisent : " Je ne citerai pas les bouffonnes histoires des écoles roulantes qui suivaient les tribus et qui les mettaient en fuite comme l'apparition d'une panthère fait courir un troupeau de moutons » (B.E. février $1896: 26$ ).

9. D'autres lettres indiquent les prix du mobilier et du matériel scolaire (20.1.3 et 16.10.1896).

10. Le décret du 18 octobre 1892 avait établi quatre catégories d'écoles pour l'enseignement des indigènes : principales (au moins 3 classes), élémentaires (moins de 3 classes), préparatoires (une seule classe), enfantines.

11. Institués par le décret de 1883, l'un annexé à l'école normale d'Alger, l'autre à celle de Constantine, créées respectivement en 1865 et 1879. Ils furent abrogés en 1924 (D.a. 1946).

12. MM. Medouhès et Ben Ali (B.E. janvier 1894, 29.9.1896). 
13. Sous la conduite, au début, d'E. Scheer, le premier inspecteur des écoles primaires indigènes en Algérie. Le Plan déclarait : «Les Arabes seuls qui ont vu la France en connaissent la beauté et la richesse ; et, dans leur langage pittoresque, la comparent à un immense jardin. Cette impression est la vraie, et elle devrait ressortir des leçons de géographie » (Plan 1890 : 83). M. Testas passe ses vacances à Marnaves dans le Tarn (3.8.1895).

14. Le salaire d'un instituteur français en Algérie, plus élevé d'environ un quart par rapport à celui de la Métropole, pouvait inciter à s'exiler dans une oasis lointaine. Les instituteurs des écoles indigènes bénéficiaient de plusieurs indemnités particulières. M. Testas est instituteur stagiaire en congé au moment de sa nomination à Négrine, le 1er octobre 1892. Sa femme, exinstitutrice, est nommée stagiaire adjointe, en septembre 1894, création d'emploi. Ils seront nommés à Chateaudun-du-Rhummel, près de Sétif, en 1906 (B.E. juillet 1906).

15. MM. Medouhès et Labrache sont chargés respectivement, le 23 septembre 1896, emplois nouveaux, des écoles nomades des Brarcha et des Allaouna, créée le 16 octobre 1896 (29.9.1896). M. Ben Ali succédera au premier le 28 octobre 1896 (B.E. novembre 1896). M. Testas enseignera l'arabe après avoir passé le brevet de cette langue en 1896 : «par ordre de l'autorité académique, je suis chargé d'enseigner la langue arabe conjointement au français (...). Dès le 20, 35 élèves recevront mes leçons de grammaire d'arabe vulgaire » (19.4.1896) ; « tous les élèves qui suivent mes cours savent les lettres de l'alphabet. Méthode : celle de Machuel» (1.5.1896); « le programme du mois a compris la formation de mots, l'étude des voyelles et des lettres qui n'ont pas de liaison avec les autres. Dictées et lectures » (28.5.1896).

16. «L'Arabe n'entre dans l'enseignement que poussé par le besoin. Il préfère le métier de gardechampêtre, cavalier de l'administrateur, chaouch car il a un fusil et un cheval et le prestige de l'uniforme. Il laisse l'enseignement dès qu'il trouve une situation qui flatte plus son amourpropre " (Mourlan 1903 : 113). M. Medouhès continuera à enseigner. Il sera nommé à Tiget en 1898 (B.E. novembre 1898).

17. II va jusqu'à demander au chef militaire s'il doit fournir " même en vacances, les renseignements trimestriels sur les maladies »! (6.7.1897).

18. «Je vous suis très reconnaissant des peines et dérangements que l'établissement et le fonctionnement de cette nouvelle école vous ont occasionnés cette année »(10.4.1896).

19. Tableaux des fréquentations tels qu'ils sont transcrits par les maîtres : de gauche à droite Négrine, mai 1895 (57.5.7S95) et Allaouna, janvier 1897 (31.1.1897)

\begin{tabular}{|c|c|c|c|c|}
\hline & $\begin{array}{l}I^{\text {eret }} \\
\text { classe }\end{array}$ & $\begin{array}{l}2^{e} \\
\text { classe }\end{array}$ & $\begin{array}{l}\text { total } \\
\text { Négrine }\end{array}$ & $\begin{array}{l}I^{\text {ére }} \text { classe } \\
\text { Allaouna }\end{array}$ \\
\hline $\begin{array}{l}\text { élèves inscrits depuis } \\
\text { le ler octobre }\end{array}$ & & & & 24 \\
\hline $\begin{array}{l}\text { élèves inscrits au } \\
\text { registre d'appel }\end{array}$ & 29 & 30 & 59 & 24 \\
\hline $\begin{array}{l}\text { maximum des } \\
\text { présences possibles } \\
\text { par } 1 / 2 \text { jour de classe }\end{array}$ & 1276 & 1320 & 2596 & 696 \\
\hline $\begin{array}{l}\text { absences par } 1 / 2 \text { jour } \\
\text { de classe }\end{array}$ & 296 & 161 & 457 & 4 \\
\hline $\begin{array}{l}\text { présences effectives } \\
\text { par } 1 / 2 \text { jour de classe }\end{array}$ & 980 & 1159 & 2139 & 692 \\
\hline $\begin{array}{l}\text { nombre de } 1 / 2 \text { jours } \\
\text { de classe }\end{array}$ & & 44 & & 32 \\
\hline
\end{tabular}

Figurent les tableaux de M. Testas pour 1894-95 (de décembre 1894 à mai 1895), 1895-96, 1896-97. Pour M. Ben Ali : de janvier 1897 à juin 1897 et pour les mois de novembre et décembre, non datés. Pour M. Labrache : de janvier 1897 à juin 1897. 
20. Le décret de 1892 ne pose pas en principe l'obligation scolaire pour les indigènes, ne concernant que les garçons. La non fréquentation faisait encourir des peines aux parents, renforcées par loi du 21 décembre 1897.

21. Devant la mauvaise volonté du khalifa à fournir à $\mathrm{M}$. Labrache des bêtes de somme pour son ravitaillement, le commandant est forcé de constater : « quand le caïd est présent, l'instituteur obtient ce dont il a besoin » (3.6.1897).

22. Le paludisme surtout, d'où la requête de $M$. Testas au commandant de quinine pour sa femme et lui, le cheikh, la population indigène (13.2.1896). Malades en mai l'instituteur et sa femme, atteinte par la coqueluche et absente du 24 janvier au 4 février 1895 (1.2.1895), sont à nouveau victimes de fièvres du 10 octobre au 15 novembre (20.1.1896). Ils obtiennent une prolongation des vacances jusqu'au 15 octobre ; l'école sera fermée du 7 février au 4 mars 1895 à la suite de l'épidémie de coqueluche.

23. M. Testas occupe alors ses élèves en leur apprenant la fabrication du pain qui, vendu à des touristes, procure un bénéfice de $25 \mathrm{fr}$ (B.E. février 1895).

24. En janvier 1897, il n'a plus que 15 élèves sur les 24 inscrits en octobre (31.1.1897) ; « 11 élèves ne sont pas sûrs » écrit-il encore en mars (2.3.1897).

25. M. Ben Ali n'a reçu qu'une partie des fournitures scolaires. Il lui manque ce dont il a le plus besoin, crayons d'ardoise, cahiers d'écriture, cahiers de devoirs : " je me trouve dans l'impossibilité de continuer à faire régulièrement la classe à défaut de ces objets » (19.2, 15.3.1897). L'adjoint des Allaouna renvoie du matériel détérioré : le trépied du tableau noir, le pliant chaise et le réveil en en recommandant la réparation le plus tôt possible car « ces objets sont d'usage fréquent pour l'école » (14.5.1897). Il réitère sa demande le 2 juin (2.6.1897).

26. A rédiger en trois exemplaires, précise $M$. Labrache : pour la commune, l'inspection primaire, les archives de l'école (27.10.1896).

27. Le B.E. février 1895, mentionne l'initiative de M. Testas.

28. Cf. L. Machuel, cité dans les listes des livres : il prônait une méthode de lecture et aussi d'apprentissage du langage pour des élèves d'origine hétérogène pratiquant peu le français en dehors de l'école (Machuel 1885).

29. Un instituteur constate " qu'il manque un complément à l'œuvre de scolarisation des indigènes qui date de six ans que rien ou presque rien n'est venu perfectionner (...). Vous avez déploré l'inappropriation à votre école des manuels scolaires en vogue, le manque absolu de livres édités spécialement pour les petits indigènes... hélas, auteurs et éditeurs se tiennent sur la réserve, attendant pour dépenser leurs veilles et leurs capitaux, que nous puissions leur assurer 50.000 acheteurs ", (B.E. août 1898 : 34). Les programmes scolaires ne seront unifiés qu'entre 1945 et 1949 .

30. Le cours élémentaire prévoyait $1 \mathrm{~h}$. de travail manuel trois jours par semaine : travail du bois, du fer, de la maçonnerie (Plan 1890). Le cahier du corpus contient cinq dessins. La lettre de l'inspecteur primaire de Batna au commandant mentionnait qu'il joignait, à l'envoi du cahier de l'école de Négrine, des «travaux de l'école des Brarcha » (15.7.1897).

31. «Pour inculquer à n'importe quel peuple ce que nous appelons notre civilisation, il faut peut-être commencer par lui donner les mêmes besoins matériels que nous, éveiller en lui les mêmes idées de bien-être, de confort, de propreté et de lignes régulières » (Rambaud 1910 : 53 ).

32. Cf. Besserve-Bernollin, etc. 1981.

33. Le Plan de 1898 accentuera cette tendance. 


\section{RÉSUMÉS}

L'enseignement $\mathrm{du}$ français dans les "écoles indigènes " $\mathrm{du}$ sud-est algérien après la réorganisation de l'enseignement primaire, à travers la correspondance entre instituteurs français et indigènes et leurs supérieurs administratifs, de 1895 à 1897.

Based on the correspondence from 1895 to 1897 between both French and Algerian teachers and the authorities, the teaching of the French language in « Algerian schools " in the South East of the country, following the reforms adopted by primary schools is examined.

INDEX

Mots-clés : Algérie, , enseignement primaire, instituteurs, correspondance

Keywords : Algeria, , primary schools, teachers, correspondence

\section{AUTEUR}

CLAUDE BISQUERRA

Université de L'Aquila 